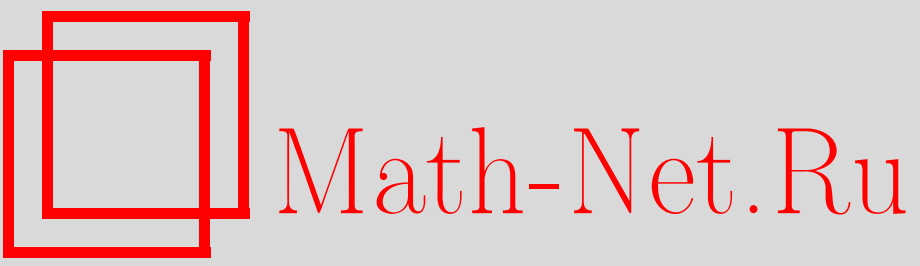

А. Г. Елисеев, Т. А. Ратникова, Асимптотическое решение сингулярно возмущенной задачи Коши при наличии рациональной «простой» точки поворота, Итоги науки и техн. Сер. Соврем. мат. и ее прил. Темат. обз., 2021, том 190, 81-87

DOI: https://doi.org/10.36535/0233-6723-2021-190-81-87

Использование Общероссийского математического портала Math-Net.Ru подразумевает, что вы прочитали и согласны с пользовательским соглашением

http://www.mathnet.ru/rus/agreement

Параметры загрузки:

IP : 3.85 .183 .62

26 апреля 2023 г., 17:53:28 


\title{
АСИМПТОТИЧЕСКОЕ РЕШЕНИЕ СИНГУЛЯРНО ВОЗМУЩЕННОЙ ЗАДАЧИ КОШИ ПРИ НАЛИЧИИ РАЦИОНАЛЬНОЙ «ПРОСТОЙ» ТОЧКИ ПОВОРОТА
}

\author{
(c) 2021 г. $\quad$ А. Г. ЕЛИСЕЕВ, Т. А. РАТНИКОВА
}

\begin{abstract}
АннотАция. В статье на основе метода регуляризации С. А. Ломова построено асимптотическое решение сингулярно возмущенной задачи Коши в случае нарушения условий стабильности спектра предельного оператора. В частности, рассмотрена задача с «простой» точкой поворота, т.е. одно собственное значение обращается в ноль при $t=0$ и имеет вид $t^{m / n}$ (предельный оператор дискретно необратим).
\end{abstract}

Ключевъе слова: сингулярно возмущенная задача Коши, асимптотическое решение, метод регуляризации, точка поворота.

\section{ASYMPTOTIC SOLUTION}

\section{OF A SINGULARLY PERTURBED CAUCHY PROBLEM}

\section{IN THE PRESENCE OF A RATIONAL "SIMPLE" TURNING POINT}

\author{
(C) 2021 A. G. ELISEEV, T. A. RATNIKOVA
}

\begin{abstract}
In this paper, based on S. A. Lomov's regularization method, we construct an asymptotic solution of a singularly perturbed Cauchy problem in the case of violation of the stability conditions for the spectrum of the limit operator. In particular, we consider the problem with a "simple" turning point, i.e., where one eigenvalue vanishes for $t=0$ and has the form $t^{m / n}$ (the limit operator is discretely irreversible).
\end{abstract}

Keywords and phrases: singularly perturbed Cauchy problem, asymptotic solution, regularization method, turning point.

AMS Subject Classification: 34E20

В данной работе методом регуляризации С. А. Ломова (см. [1]) строится асимптотическое решение задачи Коши в случае рациональной «простой» точки поворота. Метод регуляризации позволяет построить равномерное на всем отрезке $[0, T]$ асимптотическое решение, а при дополнительных условиях на параметры сингулярно возмущенной задачи и ее правую часть - точное решение. Идея данной работы восходит к работе [2], в которой разработаны методы решения сингулярно возмущенной задачи Коши в случае «простой» точки поворота предельного оператора с натуральным показателем.

Поясним термин «простая» точка поворота. Пусть дана задача Коши

$$
\left\{\begin{array}{l}
\varepsilon u^{\prime}=A(t) u+h(t), \\
u(0, \varepsilon)=u^{0}
\end{array}\right.
$$

и выполнены следующие условия: 
(i) $h(t) \in C^{\infty}\left([0, T], R^{n}\right)$;

(ii) $A(t) \in C^{\infty}\left([0, T], L\left(R^{n}, R^{n}\right)\right)$;

(iii) спектр оператора $A(t)$ удовлетворяет условиям:

(a) $\lambda_{i}(t) \neq \lambda_{j}(t) \forall i \neq j, i, j=\overline{1, n}$;

(b) $\lambda_{i}(t) \neq 0, i=\overline{2, n}$;

(c) условие «простой» точки поворота с натуральным показателем: $\lambda_{1}(t)$ имеет нуль $k$-порядка, т.е. $\lambda_{1}(t)=t^{k} a(t)$ для всех $t \in[0, T]$, где $a(t) \neq 0$.

Следует отметить, что термин «простая» точка поворота был предложен автором метода регуляризации С. А. Ломовым в [2].

1. Формализм метода регуляризации. Точка $\varepsilon=0$ для задачи (1) является особой в том смысле, что классические теоремы существования решения задачи Коши не имеют места. Поэтому в решении этой задачи возникают существенно особые сингулярности. При выполнении условия стабильности для спектра $A(t)$ существенно особые сингулярности описываются с помощью экспонент вида:

$$
e^{\varphi_{i}(t) / \varepsilon}, \quad \varphi_{i}(t)=\int_{0}^{t} \lambda_{i}(s) d s, \quad i=\overline{1, n},
$$

где $\varphi_{i}(t)$ - гладкая (в общем случае комплексная) функция действительного переменного $t$. Для решения линейных однородных уравнений такие сингулярности были описаны еще Лиувиллем (см. [3]).

Если же условия стабильности нарушены хотя бы для одной точки спектра оператора $A(t)$ (условие іiіс), то кроме экспоненциально существенно особых сингулярностей в решении неоднородного уравнения возникают еще и сингулярности вида:

$$
\sigma_{i}=e^{\varphi_{1}(t) / \varepsilon} \int_{0}^{t} e^{-\varphi_{1}(s) / \varepsilon} s^{i} d s, \quad i=\overline{0, k-1},
$$

которые при $\varepsilon \rightarrow 0$ имеют степенной характер убывания при соответствующих ограничениях на $\lambda_{1}(t)$, при этом предполагается, что остальные точки спектра не обращаются в нуль при $t=0$ (условия iiia, iiib).

Сингулярно возмущенные задачи возникают обычно в случаях, когда область определения исходного оператора, зависящего от $\varepsilon$ при $\varepsilon \neq 0$, не совпадает с областью определения предельного оператора при $\varepsilon=0$. При изучении задач с «простой» точкой поворота возникают дополнительные условия, когда область значений исходного оператора не совпадает с областью значений предельного оператора.

Для того, чтобы выявить особенности построения решения в случае рациональной точки поворота, и не усложнять изложение материала дополнительными вычислениями при наличии стабильной части спектра, рассмотрим следующую задачу Коши:

$$
\left\{\begin{array}{l}
\varepsilon u^{\prime}+t^{m / n} u=h(t), \\
u(0, \varepsilon)=u^{0}
\end{array}\right.
$$

и выполнены следующие условия:

(i) $h(t) \in C^{\infty}([0, T], R)$;

(ii) $m, n \in \mathbb{N}$.

Заменой переменной $\tau=t^{1 / n}$ задача (2) сводится к задаче

$$
\left\{\begin{array}{l}
\varepsilon u^{\prime}(\tau, \varepsilon)+\tau^{p} u(\tau, \varepsilon)=\tau^{n-1} h\left(\tau^{n}\right), \\
u(0, \varepsilon)=u^{0}, \quad \text { где } m+n-1=p,
\end{array}\right.
$$

методы решения которой изложены в [2].

В данной задаче на структуру решения (3) сильно влияет правая часть уравнения (так как она не принадлежит области значений предельного оператора). 
Основные сингулярности данной задачи [2] имеют вид

$$
e^{-\varphi(\tau) / \varepsilon} ; \quad \sigma_{i}=e^{-\varphi(\tau) / \varepsilon} \int_{0}^{\tau} e^{\varphi(s) / \varepsilon} s^{i} d s, \quad i=\overline{0, p-1},
$$

где $\varphi(\tau)=\tau^{p+1} /(p+1)$.

Согласно методу регуляризации в случае «простой» точки поворота решение ищется в виде

$$
u(\tau, \varepsilon)=\sum_{k=-1}^{\infty} \varepsilon^{k} u_{k}=e^{-\varphi(\tau) / \varepsilon} x(\tau, \varepsilon)+\sum_{i=0}^{p-1} y^{i}(\tau, \varepsilon) \sigma_{i}+z(\tau, \varepsilon),
$$

где $x(\tau, \varepsilon), y^{i}(\tau, \varepsilon), z(\tau, \varepsilon), i=\overline{0, p-1}$, - гладкие по $\tau$ функции, степенным образом зависящие от $\varepsilon$.

Подставляя (4) в задачу (3) и выделяя слагаемые при одинаковых сингулярностях, получим следующую задачу:

$$
\left\{\begin{array}{l}
(x(\tau, \varepsilon))^{\prime}=0, \\
\left(y^{i}(\tau, \varepsilon)\right)^{\prime}=0, \quad i=\overline{0, p-1} \\
\tau^{p} z(\tau, \varepsilon)=\tau^{n-1} h\left(\tau^{n}\right)-\varepsilon\left[y^{0}(\tau, \varepsilon)+\tau y^{1}(\tau, \varepsilon)+\ldots+\tau^{p-1} y^{p-1}(\tau, \varepsilon)\right]-\varepsilon z^{\prime}(\tau, \varepsilon), \\
x(0, \varepsilon)+z(0, \varepsilon)=u^{0}
\end{array}\right.
$$

Функции $y^{i}(\tau, \varepsilon)$ не участвуют в начальном условии системы $(5)$, так как $\sigma_{i}(0, \varepsilon)=0$.

Задача (5) является регулярной по степеням $\varepsilon$, поэтому, разлагая функции $x, y^{i}, z$ в ряды по степеням $\varepsilon$ :

$$
\left\{\begin{array}{l}
x=\sum_{k=-1}^{\infty} \varepsilon^{k} x_{k}(\tau) \\
y^{i}=\sum_{k=-1}^{\infty} \varepsilon^{k} y_{k}^{i}(\tau), \quad i=\overline{0, p-1} \\
z=\sum_{k=-1}^{\infty} \varepsilon^{k} z_{k}(\tau)
\end{array}\right.
$$

и приравнивая выражения при одинаковых степенях $\varepsilon$, получим серию итерационных задач:

$$
\left\{\begin{array}{l}
x_{k}^{\prime}(\tau)=0, \\
\left(y_{k}^{i}(\tau)\right)^{\prime}=0, \quad i=\overline{0, p-1} \\
\tau^{p} z_{k}(\tau)=-z_{k-1}^{\prime}(\tau)-\sum_{i=0}^{p-1} \tau^{i} y_{k-1}^{i}(\tau)+\delta_{0}^{k} \tau^{n-1} h\left(\tau^{n}\right) \\
x_{k}(0)+z_{k}(0)=\delta_{0}^{k} u^{0}, \quad k=\overline{-1, \infty}
\end{array}\right.
$$

где $\delta_{0}^{k}$ - символ Кронекера.

Отрицательная степень по $\varepsilon$ возникает из-за того, что $\tau^{n-1} h\left(\tau^{n}\right)$ не принадлежит области значений предельного оператора.

Для решения итерационных задач (7) сформулируем теорему о точечной разрешимости уравнения

$$
\tau^{p} z(\tau)=\tau^{n-s} h\left(\tau^{n}\right)
$$

где $s$ - любое натуральное фиксированное число, $0 \leqslant s \leqslant n-1$.

Теорема 1. Пусть дано уравнение

$$
\tau^{p} z(\tau)=\tau^{n-s} h\left(\tau^{n}\right), \quad m+n-1=p,
$$


и выполнены условия (i) и (ii) задачи (2). Тогда уравнение (8) разрешимо в классе гладких функияй тогда и только тогда, когда

$$
h^{k}(0)=0, \quad k=\overline{0,\left[\frac{m+s-1}{n}\right]} .
$$

Доказательство. Необходимость. Пусть уравнение (8) разрешимо. Тогда

$$
z(\tau)=\tau^{n-s-p} h\left(\tau^{n}\right)
$$

Разложим $h\left(\tau^{n}\right)$ по формуле Маклорена

$$
z(\tau)=\sum_{k=0}^{N} \frac{h^{(k)}(0)}{k !} \tau^{(k+1) n-p-s}+o\left(\tau^{(N+1) n-p-s}\right) .
$$

Из гладкости решения (9) с необходимостью следует, что

$$
(N+1) n-s \leqslant p<(N+2) n-s .
$$

Учитывая, что $p=m+n-1$, получим

$$
N-\frac{s-1}{n} \leqslant \frac{m}{n}<(N+1)-\frac{s-1}{n} .
$$

Отсюда $N=\left[\frac{m+s-1}{n}\right]$ и $h^{k}(0)=0, k=\overline{0, N}$.

Достаточность очевидна.

Решение (8) при выполнении условий теоремы запишется в виде

$$
z(\tau)=\tau^{n-\nu} h_{1}\left(\tau^{n}\right)
$$

где $0 \leqslant \nu \leqslant n-1, h_{1}(0) \neq 0$.

Замечание. При решении итерационных задач при определении частного решения $z_{k}(\tau)$ приходится дифференцировать выражение $\tau^{n-\nu} h_{1}\left(\tau^{n}\right)$ :

$$
\frac{d}{d \tau} \tau^{n-\nu} h_{1}\left(\tau^{n}\right)=(n-\nu) \tau^{n-\nu-1} h_{1}\left(\tau^{n}\right)+n \tau^{2 n-\nu-1} h_{1}\left(\tau^{n}\right)=\tau^{n-\nu-1} h_{2}\left(\tau^{n}\right) .
$$

Таким образом, через $(n-1)$ шагов получим:

$$
\frac{d^{n-1}}{d \tau^{n-1}} \tau^{n-1} h\left(\tau^{n}\right)=h_{1}\left(\tau^{n}\right)
$$

Рассмотрим систему (7) при $k=-1$ :

$$
\left\{\begin{array}{l}
x_{-1}^{\prime}(\tau)=0 \\
\left(y_{-1}^{i}(\tau)\right)^{\prime}=0, \quad i=\overline{0, p-1} \\
\tau^{p} z_{-1}(\tau)=0 \\
x_{-1}(0)+z_{-1}(0)=0
\end{array}\right.
$$

Решение системы (8) запишется в виде

$$
\left\{\begin{array}{l}
x_{-1}(\tau)=x_{-1}(0)=0 \\
y_{-1}^{i}(\tau)=y_{-1}^{i}(0), \quad i=\overline{0, p-1} \\
z_{-1}(\tau)=0
\end{array}\right.
$$


На данном итерационном шаге $(k=-1) y_{-1}^{i}(0)$ - произвольные числа, которые определяются из условия точечной разрешимости при $k=0$ :

$$
\left\{\begin{array}{l}
x_{0}^{\prime}(\tau)=0, \\
\left(y_{0}^{i}(\tau)\right)^{\prime}=0, \quad i=\overline{0, p-1} \\
\tau^{p} z_{0}(\tau)=-z_{-1}^{\prime}(\tau)-\sum_{i=0}^{p-1} \tau^{i} y_{-1}^{i}(0)+\tau^{n-1} h\left(\tau^{n}\right) \\
x_{0}(0)+z_{0}(0)=u^{0}
\end{array}\right.
$$

Решение задачи (13) запишется как $x_{0}(\tau)=x_{0}(0), y_{0}^{i}(\tau)=y_{0}^{i}(0), i=\overline{0, p-1}$, которые определяются из условия разрешимости уравнения

$$
\tau^{p} z_{0}(\tau)=\tau^{n-1} h\left(\tau^{n}\right)-\sum_{i=0}^{p-1} \tau^{i} y_{-1}^{i}(0)
$$

и начальных условий.

На основании теоремы разрешимости следует, что

где

$$
y_{-1}^{i}(0)=\frac{h^{j}(0)}{j !}
$$

$$
\operatorname{ord}\left(\tau^{i}\right)=\operatorname{ord}\left(\tau^{n(j+1)-1}\right), \quad i=\overline{0, p-1}, \quad j=\overline{0, N}, \quad N=\left[\frac{m}{n}\right] .
$$

$\operatorname{ord}\left(\tau^{i}\right)=i$ означает степень $\tau^{i}$.

Остальные $y_{-1}^{i}(0)=0$. Решение $z_{0}(\tau)$ запишется в виде

$$
z_{0}(\tau)=\tau^{n(N+2)-p-1} h_{0}\left(\tau^{n}\right)=\tau^{s} h_{0}\left(\tau^{n}\right)
$$

где $s$-целое число, $s \in[0, n-1]$. Величина $x_{0}(0)$ определяется из начальных условий $x_{0}(0)+$ $z_{0}(0)=u^{0}$. Тогда

$$
x_{0}(0)= \begin{cases}u^{0}, & \text { если } z_{0}(0)=0, \\ u^{0}-z_{0}(0), & \text { если } z_{0}(0) \neq 0 .\end{cases}
$$

Таким образом, решение на «-1» шаге определено:

$$
u_{-1}(\tau, \varepsilon)=\sum_{i=0}^{p-1} \frac{h^{j}(0)}{j !} \sigma_{i},
$$

где суммирование ведется по слагаемым, удовлетворяющих условию

$$
\operatorname{ord}\left(\tau^{i}\right)=\operatorname{ord}\left(\tau^{n(j+1)-1}\right) .
$$

Чтобы определить $y_{0}^{i}(\tau)$, рассмотрим итерационную систему на шаге $k=1$ :

$$
\left\{\begin{array}{l}
x_{1}^{\prime}(\tau)=0, \\
\left(y_{1}^{i}(\tau)\right)^{\prime}=0, \quad i=\overline{0, p-1} \\
\tau^{p} z_{1}(\tau)=-z_{0}^{\prime}(\tau)-\sum_{i=0}^{p-1} \tau^{i} y_{0}^{i}(0) \\
x_{0}(0)+z_{0}(0)=0 .
\end{array}\right.
$$

Рассмотрим уравнение относительно $z_{1}(\tau)$. Согласно замечанию

$$
\tau^{p} z_{1}(\tau)=-\tau^{s-1} h_{0}\left(\tau^{n}\right)-\sum_{i=0}^{p-1} \tau^{i} y_{0}^{i}(0),
$$

где $h_{0}\left(\tau^{n}\right)$ - некоторая гладкая функция, $h_{0}(0) \neq 0$. 
На основании теоремы разрешимости имеем $y_{0}^{i}(0)=h_{0}^{(j)}(0) / j$ !, для которых

$$
\operatorname{ord}\left(\tau^{i}\right)=\operatorname{ord}(n j+s-1), \quad i=\overline{0, p-1}, \quad j=\overline{0, N}, \text { где } N=\left[\frac{m-s}{n}\right] .
$$

Отсюда $z_{1}(\tau)=\tau^{n-q} h_{1}\left(\tau^{n}\right), 0 \leqslant q \leqslant n-1$ и

$$
x_{0}(0)=\left[\begin{array}{ll}
0, & \text { если } z_{1}(0)=0, \\
-z_{1}(0), & \text { если } z_{1}(0) \neq 0 .
\end{array}\right.
$$

Следовательно, на нулевом итерационном шаге полностью находится главный член асимптотики:

$$
u_{\text {гл }}(\tau, \varepsilon)=\frac{1}{\varepsilon} \sum_{i=0}^{p-1} \frac{h^{j}(0)}{j !} \sigma_{i}+x_{0}(0) e^{-\varphi(\tau) / \varepsilon}+\sum_{i=0}^{p-1} y_{0}^{i}(0) \sigma_{i},
$$

где в первом слагаемом суммирование ведется по условию (15), в третьем слагаемом суммирование ведется по условию (18).

Аналогично можно найти любой член асимптотического решения.

2. Оценка асимптотического члена. Пусть

$$
u(\tau, \varepsilon)=\sum_{k=-1}^{N} \varepsilon^{k} u_{k}(\tau, \varepsilon)+\varepsilon^{N+1} R_{N}(\tau, \varepsilon),
$$

где $u_{k}(\tau, \varepsilon)$ - решения итерационных задач. Подставим уравнение (20) в задачу (3). Тогда получим задачу Коши:

$$
\left\{\begin{array}{l}
\varepsilon R_{n}^{\prime}(\tau, \varepsilon)+\tau^{p} R_{n}(\tau, \varepsilon)=H(\tau) \\
R_{n}(0, \varepsilon)=0
\end{array}\right.
$$

Здесь

$$
H(\tau)=\tau^{p} z_{N+1}+z_{N}^{\prime}+\sum_{i=0}^{p-1} \tau^{i} y_{N}^{i}(0)
$$

удовлетворяет условию точечной разрешимости. Тогда, оценивая $R_{n}(\tau, \varepsilon)$ по норме $C[0, T]$, получим оценку $\left\|R_{n}(\tau, \varepsilon)\right\| \leqslant C$.

Теорема 2. Пусть задана задача (3) и выполнены условия (i) и (ii). $C>0$-константа, не зависящая от $\varepsilon$. Тогда верна оченка

$$
\left\|u(\tau, \varepsilon)-\sum_{k=-1}^{N} \varepsilon^{k} u_{k}(\tau, \varepsilon)\right\| \leqslant C \varepsilon^{N+1} .
$$

Теорема 3. Пусть выполнены условия теоремы 2 и дополнительные условия точечной разрешимости $h^{i}(0)=0, i=\overline{0, k}, k=[\mathrm{m} / n]$. Тогда

$$
\lim _{\varepsilon \rightarrow 0} u(\tau, \varepsilon)=u_{0}(\tau), \quad \text { где } \quad u_{0}(\tau)=\frac{\tau^{n-1} h\left(\tau^{n-1}\right)}{\tau^{p}}=\tau^{n-s} h_{1}\left(\tau^{n}\right) .
$$

Здесъ $h_{1}(0) \neq 0,0 \leqslant s \leqslant n-1$, или - в терминах исходной задачи $-u_{0}(t)=t^{(1-s / n)} h_{1}(t)$.

3. Примеры. В заключение приведем два примера.

\section{Пример 1.}

$$
\left\{\begin{array}{l}
\varepsilon u^{\prime}+t^{3 / 2} u=h(t) \\
u(0, \varepsilon)=u^{0}, \quad t \in[0, T]
\end{array}\right.
$$

Регуляризирующие функции в данном случае будут

$$
e^{-2 t^{5 / 2} / 5 \varepsilon} ; \quad \sigma_{i}=e^{-2 t^{5 / 2} / 5 \varepsilon} \int_{0}^{\sqrt{t}} e^{2 s^{5 / 2} / 5 \varepsilon} s^{i} d s, \quad i=\overline{0,3} .
$$


Главный член асимптотики решения задачи запишется в виде

где

$$
\begin{aligned}
u=e^{-2 t^{5 / 2} / 5 \varepsilon} u^{0}+\sigma_{0}\left(-z_{0}^{\prime}(0)\right)+ & \sigma_{1}\left(\frac{h(0)}{\varepsilon}\right)+ \\
& +\sigma_{2}\left(-z_{0}^{\prime \prime}(0)\right)+\sigma_{3}\left(\frac{h^{\prime}(0)}{\varepsilon}\right)+\frac{\left(h(t)-h(0)-h^{\prime}(0) t\right) \sqrt{t}}{t^{2}},
\end{aligned}
$$

$$
z_{0}=\frac{\left(h(t)-h(0)-h^{\prime}(0) t\right) \sqrt{t}}{t^{2}} .
$$

\section{Пример 2.}

$$
\left\{\begin{array}{r}
\varepsilon u^{\prime}+t^{1 / 2} u=h(t), \\
u(0, \varepsilon)=u^{0}, \quad t \in[0, T] .
\end{array}\right.
$$

Регуляризирующие функции в данном случае будут

$$
e^{-2 t^{3 / 2} / 3 \varepsilon} ; \quad \sigma_{i}=e^{-2 t^{3 / 2} / 3 \varepsilon} \int_{0}^{\sqrt{t}} e^{2 s^{3 / 2} / 3 \varepsilon} s^{i} d s, \quad i=0,1 .
$$

Главный член асимптотики решения задачи запишется в виде

$$
u=e^{-2 t^{3 / 2} / 3 \varepsilon} u^{0}+\sigma_{0}\left(-h_{0}^{\prime}(0)\right)+\sigma_{1}\left(\frac{h(0)}{\varepsilon}\right)+\frac{(h(t)-h(0)) \sqrt{t}}{t} .
$$

\section{СПИСОК ЛИТЕРАТУРЫ}

1. Ломов С. А. Введение в общую теорию сингулярных возмущений. - М.: Наука, 1981.

2. Елисеев A. Г., Ломов С. А. Теория сингулярных возмущений в случае спектральных особенностей предельного оператора// Мат. сб. - 1986. - 131, № 173. - С. 544-557.

3. Lioville J. Second memoire sur le development des fonctions en series dont divers termes sont assujettis, a une meme equation// J. Math. Pure Appl. - 1837. — 2. - P. 16-35.

4. Турсунов Д. А., Кожбеков К. Г. Асимптотика решения сингулярно возмущенных дифференциальных уравнений с дробной точкой поворота// Изв. Иркутск. ун-та. - 2017. - 21. - С. 108-121.

Елисеев Александр Георгиевич

Национальный исследовательский университет

«Московский энергетический институт»

E-mail: eliseevag@mpei.ru

Ратникова Татьяна Анатольевна

Национальный исследовательский университет

«Московский энергетический институт»

E-mail: ratnikovata@mpei.ru 